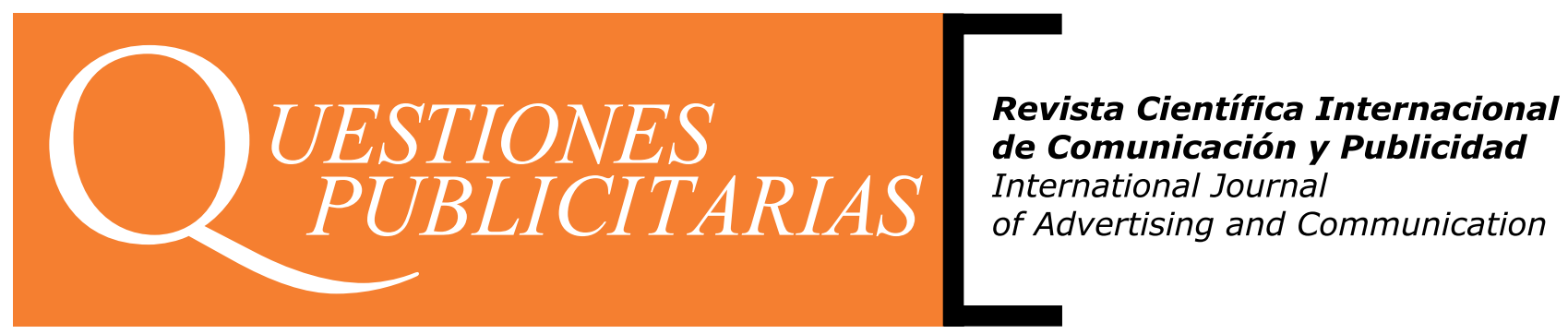

$\mathbf{V} 1 \mathbf{N} 21$

\title{
Absolutamente, Questiones Publicitarias Absolutely, Questiones Publicitarias
}

Daniel Tena-Parera, Universitat Autònoma de Barcelona, Spain.

daniel.tena@uab.cat

\begin{abstract}
Resumen: Se inicia la tercera etapa de la revista científica "Questiones Publicitarias". La Revista Científica Internacional de Publicidad y Comunicación representa un paso adelante con la adopción del sistema de edición en Open Journal System. Desde la Universitat Autónoma de Barcelona se va editar la revista decana de Publicidad en española e iberoamericana con el objetivo ser plataforma y referente para el ámbito de la Publicidad, de las Relaciones Públicas y de la Comunicación en general. Se recuperan, así, los objetivos de producción científica en un ámbito muy específico y dinámico.
\end{abstract}

Palabras clave: Questiones Publicitarias; Publicidad; Relaciones Públicas; Journal

\begin{abstract}
The third stage of journal "Questiones Publicitarias" begins. The International Journal of Advertising and Communication represents a step forward with the adoption of the editing system in the Open Journal System. From The Universitat Autónoma de Barcelona, the firts Spanish and Iberoamerican journal of Advertising will be published with the aim of being a platform and reference for the field of Advertising. Public Relations and Communication in general. Thus, the objectives of scientific production are recovered in a very specific and dynamic field.
\end{abstract}

Keywords: Questiones Publicitarias; Advertising; Public Relations; Journal

\section{EDITORIAL pp. 5 a 11}

Cómo citar este artículo / Referencia normalizada

Tena, D. (2018): “Absolutamente, Questiones Publicitarias”. Questiones Publicitarias, 1(21), p. 5-11. <https://doi.org/10.5565/rev/ qp.310> 
Era 1993 cuando "Questiones Publicitarias" (QP) publicaba sus primeras referencias. Esto la convertía en la revista científica decana del ámbito específico de la publicidad en España y del ámbito iberoamericano. La precedía "Análisis" (1989) y era seguida por "ZER" (1996), ambas más genéricas y centradas en comunicación (Balazón-Pazos et al: 2017). Ahora, QP inicia una nueva etapa que coincide con la proliferación de revistas científicas del ámbito de la comunicación. QP no renuncia a su condición como referente académico y científico, y sigue aquí, renovado, el interés que siempre la ha caracterizado: dar servicio al ámbito de la comunicación persuasiva (publicidad, relaciones públicas y comunicación en general).

Es justo agradecer, ya desde estas primeras palabras, la visión iluminada e innovadora del profesor de la Universidad de Sevilla Juan Rey cuando en 1993 publicó el primer número de QP, la revista científica internacional de Comunicación y publicidad. En aquella época no estaba sobre la mesa ni las acreditaciones, ni las urgencias en publicar, ni había una sobresaturación de nuevas cabeceras como hoy sucede. Había, eso sí, académicos e investigadores ilusionados y altruistas, con la intención de demostrar que la publicidad y las relaciones públicas merecen un interés especial en el ámbito español y, más ampliamente, iberoamericano.

Además, es justo reconocer también el trabajo, la visión y la generosidad que Juan Rey ha tenido durante años para hacernos llegar hasta hoy la realidad que representa "Questiones Publicitarias". Nuestra experiencia sobre las dificultades que pueden hoy representar la edición de una revista científica nos permite sugerir que el trayecto recorrido por QP, parece más fruto de una empecinada proposición de ayudar al ámbito de la publicidad y las relaciones públicas, que el mero ejercicio de edición. "Questiones Publicitarias" es una idea; una muy buena idea. $Y$ todos sabemos que las buenas ideas nunca se desvanecen; siempre resurgen. Así pues, vaya por delante nuestro máximo reconocimiento a los que hicieron posible aquella iniciativa en los inicios de los años noventa; y a los que han permitido hacerla llegar hasta nuestras manos durante estos años. Autores, revisores, editores y lectores... pero, reconocimiento que se materializa en Juan Rey quien, no ha dudado en encontrar una vía de pervivencia para la revista científica Internacional de Comunicación y Publicidad.

Hoy, desde la Universitat Autònoma de Barcelona, aceptamos seguir trabajando para que la decana de las revistas de publicidad en el ámbito iberoamericano perdure en el tiempo. Y en hacerlo la adaptamos a los nuevos modos de publicación. Así, publicamos en Open Journal System (OJS) que permite de manera ágil publicitar contenidos científicos online. Además, nos permite indizar los artículos y autores para que los motores de búsqueda dinamicen su impacto. Sabemos que hoy en día, el contexto de las revistas científicas se ha masificado. Pero queremos revindicar nuestro objeto de estudio: la publicidad, las relaciones públicas y la comunicación persuasiva. Y también queremos remarcar que nuestro modelo son las revistas científicas anglosajonas en el ámbito de la publicidad, que han adquirido un alto nivel de reconocimiento internacional y que, por ello, se han convertido en modelos de referencia.

\section{ARTÍCULOS CIENTÍFICOS (PAPERS), ENSAYOS Y RESEÑAS}

Esperamos que "Questiones Publicitarias" pueda continuar y aumentar, si cabe, el interés para los investigadores, académicos, profesionales y también para los estudiantes en publicidad y relaciones públicas en todas sus diversas modalidades. Para ello, la revista aceptará colaboraciones en tres secciones abiertas: artículos científicos, ensayos y reseñas.

A lo largo de su andadura la revista ha ido modificando los niveles de exigencia respecto de las aportaciones que son consideradas estrictamente artículos científicos. Para ello, adoptamos el estándar anglosajón y que es, actualmente el utilizado como referencia entre los criterios de acreditación. Sin embargo, no siempre es posible presentar una colaboración con una estructura de "paper". Entonces, la alternativa es presentarla como ensayo. Estas aportaciones, si bien, no tienen el carácter de investigación científica al uso, al no presentar la convencional estructura (fundamentación, metodología, hipótesis, averiguaciones, resultados, conclusiones), los autores pueden expresar sus opiniones, sus perspectivas, o 
incluso sus investigaciones, con un modelo más laxo pero no exento de cientifismo. Finalmente, la revista presenta la sección de reseñas, que permite hacer referencias de libros, exposiciones, tesis, o cualquier otro evento que pueda ser interesante para la comunidad científica. Todas estas aportaciones (papers, ensayos y reseñas) serán revisadas mediante el modelo de doble ciego para asegurar su idoneidad.

\section{PERFIL DEL OBJETO DE ESTUDIO}

Los límites de la investigación en publicidad y relaciones públicas han variado influenciados por las tendencias del mercado. Ciertamente a menudo se declaran nuevos objetos de estudio que, en la mayoría de los casos, no lo son; y más bien son una adaptación o la recuperación de aspectos ya preexistentes. Así, al hablar de los nuevos formatos publicitarios, de declaró, por ejemplo, al Product Placement" como tal. O nuevos perfiles profesionales como el "Community Manager" o el "Project Managers"... también son formas de actualizar las técnicas publicitarias cuando se plantean conceptos como el storytelling, la narrativa online o bien el concepto transmedia. Ciertamente es, en realidad, la recuperación, o adaptación, de tradicionales formas o perfiles de publicidad o de las relaciones públicas. QP permite poner sobre la mesa cualquier escrito o debate sobre los objetos de estudio y su vigencia, su desarrollo y estado.

\section{MULTIDISCIPLINAR, POLIÉDRICA Y MUTANTE}

La publicidad es una actividad multidisciplinar, poliédrica y mutante. Multidisciplinar dado que diversas "profesiones" influyen en el ejercicio de esta actividad: el diseño, la comunicación audiovisual, el marketing, la dirección empresarial... Poliédrica, dado que su abordaje se puede realizar desde diversas perspectivas que a menudo no permiten ver la totalidad de su identidad de manera completa. El Derecho, la Antropología, la Economía, la Producción, la Psicología, la Sociología... Y es mutante, dado que como la mayoría de las actividades vinculadas con la sociedad y los mercados, se ve obligada a una constante transformación, que, a la vez, es deseada por la publicidad y las relaciones públicas, dado que les aporta un factor esencial para su negocio: el carácter innovador.

\section{Prat Gaballí.}

Escribe Gaballí en su libro "La Publicidad Científica" que en 1850 la definición de publicidad era "el conjunto de medios destinados a dar a conocer los productos del comercio y de la industria, expresando o sin expresar ventajas particulares de marca o nombre, para venderlos casi exclusivamente en la medida de las necesidades del público" (1917: p.13). Añadiéndose años más tarde a esta definición "al mayor número posible de individuos... expresando precisamente ventajas particulares de marca o nombre de forma llamativa". Para finalmente, indicar que, en 1916, la definición más actualizada seria "ciencia que enseña a conocer el espíritu del público y a aplicar este conocimiento a los medios que disponen la imaginación u el ingenio para dar noticia al mayor número posible de individuos de la utilidad olas ventajas de un artículo, en forma que ejerza una acción eficaz, es decir, que llegue a sugerir deseos y a determinar actos de voluntad (1917: p.14).

Hoy en día, en la Ley 34/1988, de 11 de noviembre, General de Publicidad (BOE» núm. 274, de 15/11/1988) indica lo que se entiende por Publicidad: Toda forma de comunicación realizada por una persona física o jurídica, pública o privada en el ejercicio de una actividad comercial, industrial, artesanal o profesional, con el fin de promover de forma directa o indirecta la contratación de bienes muebles o inmuebles, servicios, derechos y obligaciones" $Y$ como destinatarios: Las personas a las que se dirige el mensaje publicitario o a las que éste alcance" (Título Primero: Disposiciones generales).

En realidad la profesión se ha ido gestando desde diversos conocimientos para resolver los problemas de los clientes en promocionar sus marcas y productos. Esto da lugar a diversos perfiles profesionales vinculados con la publicidad y también con las relaciones públicas. En el estricto ámbito de la Publicidad, algunos parecen evidentes: creativo/a, planer, copy, diseño y dirección de arte, producer, medios... ¿Pero qué nos depara el futuro? 


\section{Matemáticos y Filósofos}

Hace ya un par o tres de años que las agencias de Publicidad y empresas de entornos de comunicación online se están interesando en añadir a sus equipos especialistas en estadística. Es decir: matemáticos. Quién podía decir que este perfil sería una especialidad al alza hace 30 años en entornos de la comunicación persuasiva. El motivo principalmente es el "Big Data". Efectivamente, estudiar el comportamiento de la sociedad a través de su trazabilidad digital convierte al estudio de los datos en una necesidad inmediata.

Más lejos quizás, pero también muy significativo, es el fenómeno que se ha detectado en Silicon Valley. En el kilómetro cero de las tecnologías avanzadas de carácter eminentemente digital los perfiles que están sobresaliendo entre esas empresas tecnológicas es el de filósofo. Caramba!!!: filósofos en empresas dedicadas al progreso de la tecnología. Pues bien, va a resultar interesante ver cómo integran las empresas tecnológicas una visión más filosófica. Dicho de otra forma, ¿qué puede aportar la filosofía a los avances tecnológicos, a las tecnologías avanzadas que hoy en día nos envuelven? ¿Y van a estar de espalda las empresas de la comunicación persuasiva? Bien seguro que no.

Así pues, podemos aventurar que próximamente, y en la publicidad también, será necesario, por no decir que ya lo es, incorporar visiones más filosóficas, más antropológicas, a la actividad profesional.

\section{CONVERGENCIAS Y DIVERGENCAS ENTRE PUBLICIDAD Y RELACIONES PÚBLICAS}

La Publicidad y las Relaciones Públicas piensan diferente sobre los problemas que las organizaciones tienen y la forma de resolverlas. Ambas estrategias persiguen la mejora de la comunicación de las organizaciones, con el objetivo de generarles beneficios tangibles e intangibles.

Las evidencias lingüísticas introducen una complejidad a la concreción y diferenciación del objeto de estudio. Así, de la raíz latina "publicus", que aporta el significado de público (Roca \& Tena: 2013, 314); se introducen, las traducciones inglesa o francesa de los términos afines, como resultado una cierta diversificación: advertising, publicity, publicitée; public relations... Hoy en día, fruto quizás de las tendencias del mercado, aparecen nuevos conceptos que aglutinan, solapan o incluso substituyen la esencia del objeto de estudio. Así, es usual hablar de comunicación persuasiva; comunicación corporativa, empresarial u organizacional; comunicación comercial, comunicación estratégica... Por ello, parece razonable que el objeto de estudio al que "Questiones Publicitarias" debe dar respuesta sea amplio, complejo y cambiante.

Si bien existe una evidente aglutinación de los conceptos usados por los profesionales y académicos, concurre también un alto grado de especialización y diversificación. Así, podemos detectar diferencias según sea el cliente, el público al que se dirige la comunicación, el mensaje, las estrategias, el tipo de comunicación, la técnica, la relación con los medios, la forma de cobro del cliente (Roca \& Tena: 2013, 330). Todo ello da un cierto grado de ambigüedad o incluso de imprecisión terminológica, al hablar, de los aspectos vinculados a la publicidad y las relaciones públicas. Más hoy en día, que los nuevos procesos de producción online acercan los procesos de publicidad o de las relaciones públicas a neófitos del ámbito. Reivindicamos, desde aquí, que las empresas vinculadas a los ámbitos de la comunicación persuasiva incorporen en sus equipos un mayor número de empleados con estudios de publicidad y relaciones públicas.

\section{LO ESTUDIADO Y LAS TENDENCIAS FUTURAS}

Se extraen de la megainvestigación "Estudio bibliométrico sobre la investigación en publicidad en España: temáticas, investigadores, redes y centros de producción (19802015), (Balazón-Pazos et al: 2017), las líneas de investigación seguidas por los investigadores en el periodo estudiado. El $36 \%$ de las colaboraciones en las distintas revistas vinculadas al área de publicidad están destinados a "Creatividad y mensaje 
publicitario"; el $12 \%$ a la "Teoría de la publicidad y cultura"; un 10\% a "Medios publicitarios y planificación de medios"; el $8 \%$ a "Efectos de la publicidad y targets" y ya con unos porcentajes por debajo del $6 \%$ a temas como: historia de la publicidad, estructura del sistema publicitario, formatos publicitarios, profesionales de la publicidad, publicidad y educación, ética y deontología, arte y publicidad y documentación publicitaria.

Como conclusiones del trabajo mencionado (Balazón-Pazos et al: 2017), queremos destacar algunas consideraciones que nos parecen orientativas de hacía donde deben ir las investigaciones en el ámbito. Se detecta un bajo nivel de internacionalización, es decir, no existe un adecuado nivel de colaboración entre investigadores de distintas instituciones a nivel nacional y muy especialmente a nivel internacional. Tampoco se detecta una claridad en la configuración de líneas de investigación. Quizás esto se deba a la poca presencia de académicos especializados y conocedores del ámbito en la Comisión Nacional de la Actividad Investigadora, creada en 1989; ni en la Agencia Nacional de Evaluación de la Calidad (2002); que no se corrige en la descentralización de las Agencias de Calidad Universitaria en España. Según el estudio, esto provoca dos consecuencias: a) que los objetos de estudio vinculados a publicidad sean poco favorecidos en las líneas de investigación preferentes publicadas en los proyectos de investigación que optan a subvenciones; y b) los investigadores que queriendo y pudiendo ofertar estudios de investigación en concursos competitivos, no lo hagan y opten por líneas de investigación que sí reciben subvenciones.

Esta perspectiva podemos confrontarla con los objetos de estudio que se proponen en los congresos sobre publicidad y comunicación en ámbitos internacionales. Así, por ejemplo, el tema de investigación para ICORIA 2018 es la "Brand communication with multi touchpoints" y que alienta nuevos conocimientos sobre cómo la rentabilidad y el compromiso del cliente se ven afectados por múltiples mundos de contacto. Como posibles ámbitos de investigación se proponen los siguientes:

Publicidad. Responsabilidad y efectividad; Atención y procesamiento; Contenidos y Creatividad i creatividad; Aspectos interculturales; Grupos objetivos específicos; Publicidad no convencional.

Branding. Diseño; Análisis de marca; Modelos de comunicación de marca; Comunicación de marca y equidad de marca.

Gestión de la comunicación. Comunicación de marketing integrada; Relaciones públicas; Product placement, Branded content; Content marketing; Patrocinio y eventos

Consumer Behaviour. Emociones y engagement; Neurociencia y Comunicación; Construcción de redes de relación (Relationship building); Multidevice and consumer response; Personalización y hypertargeting.

Digital Media. Social Media, Mobil Media, User generated content, Touchpoints; Omniconnection and attribution; Realidad Virtual; Seguridad personal de datos en medios digitales.

Mass Media. Channel and multi-media management; Convergence management; Crossmedia strategies; Media management and media advertising.

Temas Metodológicos. Medición en Publicidad y comportamiento del consumidor; Metodologías Cualitativas en la investigación en comunicación; Metodologías Cuantitativas en la investigación en comunicación; Big data en Publicidad; Artificial neural networks, machine learning and $\mathrm{Al}$ in communication research; Métodos neurofisiológicos en la investigación en comunicación; Text, data, image analytics and Intelligent video analytics.

Así pues, con esta visión abierta a los nuevos tiempos y sin olvidarnos de los aspectos clásicos, esperamos que Questiones Publicitarias pueda continuar y aumentar, si cabe, el interés para los investigadores, para los académicos, para los profesionales y también para los estudiantes en publicidad y relaciones públicas. Especialmente poder estar atenta a las innovaciones que en su ámbito se sucedan. Para ello, la revista aceptará colaboraciones 
para las tres secciones abiertas: artículos científicos, ensayos, y reseñas. No importa el tema, importa la calidad de la colaboración. Un artículo científico fruto de una investigación subvencionada, o de una tesis doctoral, o del interés personal serán bien recibidas. Si se trata de una disertación o reflexión sobre algún tema vinculado, también será bien recibida. $\mathrm{Si}$, la aportación consiste en una reseña de un libro, de una tesis, de una exposición... sin duda que también será bien recibida. De lo que se trata es de poner en común el conocimiento que se genera entorno a la Publicidad y las Relaciones Públicas.

\section{VOLVERA EMPEZAR CON QUESTIONES PUBLICITARIAS}

En realidad, el proyecto de edición de Questiones Publicitarias es un "volver a empezar". Efectivamente, una de las condiciones que hace que una revista científica esté bien considerada es que mantenga su periodicidad. No importa tanto, la cantidad de contenido como sí su sistemática aparición pública. Este es nuestro objetivo principal en estos momentos. Volver a salir con Questiones Publicitarias. Y deseamos mantener sistemáticamente su periodicidad. La primera edición online de esta nueva etapa es la de Enero de 2018: Modesta pero ya se activa de nuevo todo el proceso de redacción, revisión, edición y finalmente publicación. Es nuestra intención volver a publicar online a finales de julio del 2018 y así, sistemáticamente cada año. Ésta va a ser nuestra pretendida secuencia: dos números online durante el año: enero y julio. Asimismo, hay que tener en cuenta que durante todo el año van a salir publicados "en prensa" aquellas aportaciones que hayan superado el proceso editorial de manera satisfactoria. Este sistema de publicación "en prensa" va a dar notoriedad lo antes posible a los contenidos de la revista y a sus autores.

Por último, como cierre de las dos ediciones online anuales, se va a publicar cada enero, a partir de 2019, una edición en papel. Dado que siempre resulta interesante para los autores disponer de su publicación en papel, se va a editar en papel un número limitado de ejemplares de la revista. Aquellos autores o instituciones, en su condición de mecenas de la revista que deseen disponer de ejemplares en papel, podrán solicitarlo y les será enviada.

Para finalizar esta editorial, no queremos olvidarnos de dos aspectos que creemos esenciales del nuevo proyecto, y que vinculan las etapas anteriores con la actual propuesta. El primer factor es la calidad de los contenidos que Questiones Publicitarias siempre ha querido promocionar. Para ello, implicamos a todos en el esfuerzo por ayudarnos con contenidos de alto valor añadido para el ámbito. El segundo factor quiere atender más bien a los detalles del ámbito. Es decir, a aquellos estudios, perspectivas o preocupaciones de los investigadores que no sean hegemónicas en la investigación pero que atienden también a los parámetros y requerimientos de la divulgación y transferencia científica. Es bien cierto que la investigación científica en Publicidad y Relaciones Públicas tiene ciertos grados de dificultad para visualizarse. $Y$ es bien cierto que los parámetros que utiliza son claros y alineados con los criterios estándares para la investigación científica. Sin embargo, la Publicidad, las Relaciones Públicas tienen grandes dosis de matices y perspectivas poco atendidas en los ámbitos más hegemónicos. Por ello, es intención del equipo editorial ser una plataforma también para aquellas aportaciones que, alejadas quizás de esos parámetros de moda, puedan permitir a los lectores comprender la amplitud y complejidad del objeto de estudio de la revista. Así pues, con la nostalgia de las etapas anteriores y alineados con el romanticismo que implica la recuperación y edición de nuevo de Questiones Publicitarias como revista científica decana de publicidad iberoamericana, esperamos poder ser útiles a la comunidad científica, profesional y académica. Por lo tanto, animamos a todos a colaborar y hacerla una revista sublime, dado que es de todos y para todos es: de la publicidad para la publicidad. 


\section{REFERENCIAS BIBLIOGRÁFICAS}

BALAZÓN-PAZOS, A.; MACHADO-PÉREZ, B.; CORREYERO-RuIZ, B. (2017). Estudio bibliométrico sobre la investigación en publicidad en España: temáticas, investigadores, redes y centros de producción (1980-2017). Revista Española de Documentación Científica 40 (2), abril-junio 2017. Doi: http://dx.doi.org/10.3989/redc.2017.2.1411

PRAT Gaballí, pedro (1917). La Publicidad Científica: Lecciones explicadas en las Clases de Enseñanza Mercantil de la Cámara de Comercio y Navegación de Barcelona. Barcelona.

RocA, D. \& TENA, D. en Rossella Gambetti \& Stephen Quigley (2013) Managing corporate communication. A cross-Cultural Approach. Palgrave Macmillan. New York.

BOE: núm. 274, de 15/11/1988

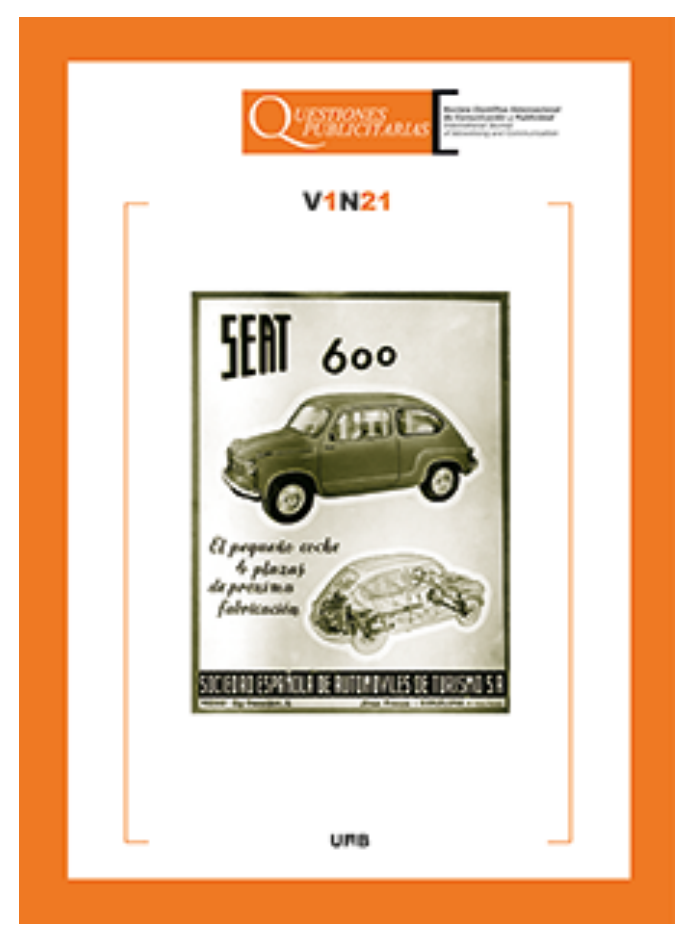

Figura 1

Cubierta de "Questiones Publicitarias" V1, N21: Composición con el Anuncio de SEAT de los años 60. (http://www.quo.es/ser-humano/veraneos: Fecha consulta: diciembre 2017). El diseño de la portada como el diseño de la revista en su conjunto, pretenden mantener la imagen visual mental de la revista QP. 